\section{Cahiers de Narratologie}

Analyse et théorie narratives

13 | 2006

Nouvelles approches de l'intertextualité

\title{
De Juan Goytisolo à Monique Lange, de Monique Lange à Juan Goytisolo : une "intertextualité conjugale"
}

\section{Emmanuel Le Vagueresse}

\section{OpenEdition}

Journals

Édition électronique

URL : http://journals.openedition.org/narratologie/340

DOI : $10.4000 /$ narratologie.340

ISSN : 1765-307X

Éditeur

LIRCES

\section{Référence électronique}

Emmanuel Le Vagueresse, « De Juan Goytisolo à Monique Lange, de Monique Lange à Juan

Goytisolo : une "intertextualité conjugale" », Cahiers de Narratologie [En ligne], 13 | 2006, mis en ligne le 01 septembre 2006, consulté le 15 novembre 2019. URL : http://journals.openedition.org/narratologie/ 340 ; DOI : 10.4000/narratologie.340

Ce document a été généré automatiquement le 15 novembre 2019.

\section{(c) (i) (9)}

Cahiers de Narratologie - Analyse et théorie narratives est mis à disposition selon les termes de la licence Creative Commons Attribution - Pas d'Utilisation Commerciale - Pas de Modification 4.0 International. 


\title{
De Juan Goytisolo à Monique Lange, de Monique Lange à Juan Goytisolo : une "intertextualité conjugale"
}

\author{
Emmanuel Le Vagueresse
}

1 Les écrivains qui parlent d'eux dans des autobiographies déguisées ou qui fictionnalisent un certain nombre d'éléments tirés de leur "vie réelle» sont certes légion. Mais une même tranche de vie racontée par deux écrivains qui forment un couple dans la vie, ou qui racontent leur histoire commune sur le mode fictionnel, est un événement bien plus rare dans la littérature. Récemment, Phillipe Sollers avec Passion fixe (Gallimard, 2000) et Dominique Rolin avec Journal amoureux (Gallimard, 2000) racontaient chacun une passion qui se révélait être la même, sous couvert de " fiction ", alors que le tout Paris des lettres savait bien sûr qui se cachait derrière les masques... C'est ce qui se produit aussi dans le cas de Juan Goytisolo (né en 1931) et Monique Lange (1926-1996), qui plus est dans deux langues et deux littératures différentes, l'espagnole et la française. On connaît mieux l'écrivain espagnol, vivant entre Paris et Marrakech, abondamment traduit chez nous et grand pourfendeur de dictatures et de mythes de tous ordres, que sa femme, récemment décédée, qui, en plus de travailler dans le milieu de l'édition et d'avoir écrit sur Cocteau et Piaf, est l'auteur d'un certain nombre de courts romans ou récits dont la plupart ont une forte résonance autobiographique et qui, en croisant justement la trajectoire de l'écrivain espagnol, établissent une réelle intertextualité dont la portée est extrêmement intéressante d'un point de vue littéraire.

2 Nous disons bien ici « littéraire » car il est lieu de préciser tout de suite qu'il ne s'agit pas pour nous de vérifier lequel des deux écrivains dit "La Vérité » sur leurs rapports dans la vie réelle, lequel des deux s'éloigne ou reste le plus fidèle par rapport à une réalité d'événements qui n'intéresseraient que le biographe. Au contraire, ce qui nous intéresse ici, c'est le traitement que chacun réserve, littérairement, textuellement et fictionnellement à un donné commun (une vie sentimentale à deux mais très anticonformiste), et comment s'établit cette intertextualité rare dans la littérature, 
d'autant que l'œuvre romanesque de Monique Lange, peu connue et, à notre connaissance, jamais étudiée comparativement par les chercheurs sur l'œuvre goytisolien - que l'on nous autorise désormais l'adjectif - quant à son apport possible à la compréhension de l'esthétique et de la thématique de l'écrivain espagnol, jette un nouvel éclairage sur le monde de «Juan sans terre ». Et cet éclairage, c'est peut-être une lumière plus nette, plus crue, démystificatrice, sur les prises de position et les obsessions de Goytisolo.

3 Pour nous livrer à l'étude de cette intertextualité, nous aurons recours aux romans ou récits suivants de Monique Lange, présentés par l'éditeur comme étant des œuvres « de fiction" mais très autobiographiques: Les platanes (1960) et Les cabines de bain (1982)1. Nous citerons également La plage espagnole (1962) lorsque les références à ce récit viendront étayer notre propos. Quant à l'œuvre de Juan Goytisolo, nous laisserons volontairement de côté Coto vedado (Chasse gardée) (1985, 1987 pour l'édition française) et En los reinos de taifa (Les royaumes déchirés) (1986, 1988 pour l'édition française), qui est présentée comme une autobiographie en deux volets, pour nous centrer sur le roman de Goytisolo qui s'occupe le plus de la relation conjugale du héros, Alvaro, avec sa compagne, Dolores, c'est-à-dire Señas de identidad (Pièces d'identité) (1966, 1968 pour l'édition française) ${ }^{2}$ ce qui nous semble plus intéressant du point de vue de l'étude d'un système de voilement/dévoilement par rapport à la réalité des rapports du couple, Alvaro/Dolores étant assez proches de Juan Goytisolo/Monique Lange. Nous citerons aussi d'autres romans ou récits de Goytisolo (particulièrement de la période des années 60 , importante à plus d'un titre, comme on le verra par la suite), lorsque ces références nous sembleront apporter des confirmations ou des précisions supplémentaires quant à cette intertextualité si particulière.

Que les amateurs de l'écrivain espagnol, peinés peut-être de ne pas trouver dans les lignes qui vont suivre des considérations sur l'intertextualité entre Goytisolo et d'autres grands noms de la littérature espagnole ou latino-américaine, de Saint Jean de la Croix à Thérèse d'Avila en passant par Góngora, mais aussi Carlos Fuentes ou Cortázar, se rassurent, ils ont à leur disposition une importante bibliographie sur ce sujet $^{3}$ ce dernier étant le plus traité depuis quelques années car ce jeu de Goytisolo avec «l'Arbre de la Littérature » dont il se dit issu est le plus pratiqué par l'auteur espagnol et donc le plus étudié en ce moment.

5 Le récit de Monique Lange qui, sans nul doute, parle le plus de ses rapports avec son écrivain de mari est Les cabines de bain (1982) et le lecteur qui connait bien Goytisolo le reconnaît à coup sûr dans cet homme dont elle évoque la vie à ses côtés lors d'une retraite en Bretagne - à Roscoff - pour se ressourcer, alors que la narratrice, qui parle à la troisième personne, semble bien être la proie d'un début de dépression. Elle revient en fait sur sa vie avec cet homme que l'on reconnaît bien comme étant Juan Goytisolo, comme dans cet extrait où elle réfléchit sur une parole de jalousie qu'il lui a dite un jour :

Qu'il est bête! Elle ne va pas dénicher un autre Espagnol qui lit le Coran, qui a toujours un manuel de grammaire arabe sur sa table de nuit, qui s'en va tous les dimanches à la même heure au hamman de la mosquée, qui aime le harira et qui écrit de beaux livres de plus en plus difficiles pour les autres et pour elle.

Lorsqu'il ne savait pas qui il était, son langage était clair. Plus il s'enfonce dans sa clarté à lui, plus ses livres deviennent sibyllins. Importants pour ses compatriotes mais où est sa patrie? Importants aussi pour quelques Arabes lettrés. Bien entendu pas pour ces hommes qu'il rencontre le dimanche $e^{4}$. 
On remarque déjà que Lange établit un lien fort chez Goytisolo, ce Juan sans terre (c'est le nom d'un de ses romans, Juan sin tierra, 1975, éd. française 1977) et sans patrie, entre découverte de soi - en fait, essentiellement, sur un plan sexuel, mais ce n'est pas dit, dans cet extrait du moins - et écriture, ce qui nous autoriserait déjà à mener notre réflexion sur la parole de Lange vers un début de critique quant à la nature réelle de ce lien : le lecteur, avec Lange, se demande pour qui Goytisolo écrit et se dit surtout qu'il n'est pas, ne peut pas être lu par ces hommes qu'il aime (on verra par la suite, dans le détail, de quel type de relations il s'agit).

Survient assez tôt sous la plume de Lange l'évocation de l'attirance, elle aussi précoce, de son compagnon pour le monde arabe, mais aussi de son attirance pour la pauvreté, la misère, via le paysage désolé d'Andalousie :

C'est au cours de son premier voyage avec elle, à Almería, qu'il a eu le choc moral et pas encore sexuel - de ce qu'il irait chercher en Afrique. (...) Les mouches de Carthagène étaient des mouches africaines. Les mouches du malheur sur les yeux des enfants qui avaient le trachome ${ }^{5}$,

8 ce que l'on retrouve chez Goytisolo dans La Chanca - du nom d'un quartier déshérité d'Almería, ville d'Andalousie - (1962, éd. française 1964), autre récit de voyage fictionnalisé publié à la même époque et parlant de ce "Gran Cáncer » qu'est la pauvreté ( «El tracoma ha devorado los ojos de los loteros que prometen 'la suerte para hoy' (...) $»^{6}$ ou Campos de Níjar (Terres de Nijar) (1960, éd. française 1964) :

El camino se abre paso a través de la gándara y Escuyos surge, de pronto, a la derecha. Es un poblado mísero, asolado por los vendavales, cuyas casas crecen sin orden ni concierto, lo mismo que hongos. No hay calles, ni siquiera veredas que merezcan tal nombre?.

Cette pauvreté, c'est ce qui fait le lien entre l'Espagne du Sud et le Maghreb, juste de l'autre côté du Détroit de Gibraltar. On retrouve un écho fictionnel de cette découverte dans le premier voyage (là encore) dans le Sud de l'Espagne d'Alvaro et de Dolores (Les Douleurs, en espagnol, ce qui montre déjà la difficulté de cette relation pour chacun des deux membres du couple) en vue du tournage d'un documentaire (car Alvaro est un photographe espagnol travaillant en France pour l'AFP sous le franquisme, dans les années 60, et vivant avec Dolores, sa compatriote) : «En tu primer contacto con el Sur la vitalidad ruda y silvestre de aquellos hombres te cautivaba (hasta el punto de irritar a Dolores) (...) »8. On en trouve un écho dans des articles ou essais aux titres éloquents comme «Tierras del Sur " (El furgón de cola, 1967, non traduit) ou dans La Chanca qui s'arrête non seulement sur la pauvreté mais aussi, ce qui "énerve Dolores", sur le regard et le corps des hommes :

Sentado en la orilla del camino, un hombre vestido con camisa de soldado, se acariciaba las mejillas, cubiertas por una barba de varios días. El pelo le cae en sortijas sobre la frente (...)

10 ou :

Victorino (...) me ayudó a meter en la cama. Se había sentado a mi lado y me miraba con una expresión vecina al amor?.

11 Et, en écho à cet homme du Sud que l'on retrouvera particulièrement à la fin de Señas de identidad:

... Tu primer contacto con el Sur fue a través de sus hombres. Desde niño los distinguías por su lenguaje y su modo de hablar, tan distinto del de los catalanes. Les oía cantar en los andamios, blasfemar en las zanjas de Obras Públicas, discutir mientras barrían las calles, pegar la hebra al sol (...) ${ }^{10}$. 
12 Déjà se fait jour le reproche d'une passion envahissante qui a à voir avec la sexualité. Mais l'on y reviendra lorsqu'il s'agira de l'étudier en détail dans les fictions principales des deux écrivains. Néanmoins, précisons d'ores et déjà que Lange reproche surtout à son compagnon de perdre toute capacité d'analyse dans les terres arabes ou musulmanes: «Lui, dont l'intelligence pouvait frôler le génie, fondait comme un loukoum sur la terre égyptienne » (p. 53). On peut citer encore :

Lorsqu'elle allait dans les pays arabes avec lui, elle le sentait possédé, envoûté. C'était comme si on lui avait jeté un sort plus fort que l'écriture, plus fort que la sexualité. Il était capable de se passionner pour les études des enfants de ses amis analphabètes, lui qui ne se souvenait peut-être pas du nombre des enfants de ses frères ${ }^{11}$.

13 Une évocation ambiguë qui n'est pas sans rappeler, dans les premières pages de Señas de identidad, son évocation à lui, Alvaro, des Noirs de Cuba :

hombría y aspereza terapéuticas que, meses atrás, habías hallado entre negros y mulatos de La Habana, perseguido tú por un amor adolescente que era como el presagio del síncope en el boulevard Richard Lenoir y de tu revocable existencia a plazo $^{12}$.

14 Il faut dire ici que cette attirance est à la fois intellectuelle et sensuelle, même si ce n'est pas encore explicite. Précisons également que la syncope du Boulevard Richard Lenoir est le moment où Alvaro prend conscience qu'il doit changer de vie et s'appliquer à une libération totale de son être, une libération que Monique Lange évoque dans ses propres lignes des Cabines de bain et dont maints exemples, cités tout au long de notre étude, donnent des preuves, comme on le constatera - en détaillant à chaque fois selon l'optique thématique en jeu.

Plus générale encore que ce goût du monde arabe, Lange évoque le goût de son compagnon pour les pauvres et les parias :

Elle se souvient d'Aubervilliers lorsqu'ils allaient manger des paellas avec des ouvriers espagnols. Elle se souvient de José qui chantait du flamenco dans le couloir du train qui les ramenait de Barcelone à Paris. « Tu vois, lui disait-il, ils emportent leur terre à la semelle de leurs souliers ». Elle ne savait pas à ce moment-là, il ne savait pas non plus lorsqu'ils traînaient dans le port à Barcelone, en buvant de la manzanilla et du jerez au Varadero, elle ne savait pas jusqu'où elle irait, jusqu'où elle serait obligée d'aller pour le comprendre ${ }^{13}$.

16 On trouve et chez Lange et chez Goytisolo toute une série de développements sur « l'amour impossible » de la narratrice avec son compagnon, et la perte des illusions sur ce «bonheur conjugal » au fur et à mesure que le temps passe, tous éléments qui sont conjugués dans une intertextualité fréquente entre ce livre et Señas de identidad, comme on va le voir : « (...) (D)ix-huit ans plus tard, ils s'aimeraient encore d'amour fou et pas seulement parce que cet amour était impossible " (Les cabines de bain, p. 122) et même : "Il avait des cousins noirs (des fils d'anciens esclaves) qui s'appelaient comme lui. C'est cela qu'il expiait. Est-ce que l'amour est une expiation ? C'est pour cela qu'il avait voulu devenir apatride»(pp.130-131), ce qui est intéressant parce que cette remarque fait le lien entre le pays ou la culture et les sentiments, la sexualité qu'on " choisit », qui est finalement le monde arabe chez Goytisolo, un peu comme dans le cas de Dolores (Monique ?) choisie par Alvaro - double de Goytisolo - puis abandonnée au profit des Arabes dans Señas de identidad, et qui donne lieu à un développement si important à la toute fin de ce roman :

aléjate de tu grey tu desvío te honra

cuanto te separa de ellos cultívalo 
lo que les molesta en ti glorifícalo

negación estricta absoluta de su orden esto eres tú ${ }^{14}$.

Physiquement, les mêmes échos sont audibles entre Señas et Les cabines de bain concernant leurs difficultés à établir des rapports physiques, mais comme toujours, par rapport à l'écrivain espagnol, le ton de la romancière française est plus franc et sans tabou:

Elle a toujours aimé les dos. Même lorsqu'elle était - ou se croyait - malheureuse, même lorsqu'ils réalisaient que plus rien n'était possible (physiquement) entre eux. Elle aimait se blottir contre lui ${ }^{15}$.

Cet amour impossible se retrouve particulièrement dans la deuxième œuvre de Lange que l'on a choisi de privilégier, Les platanes, récit (« roman » d'après le prière d'insérer Folio, en fait court récit comme La plage espagnole) qui raconte l'histoire de Claudia - par ailleurs prénom de la protagoniste de La isla (Chronique d'une île) (1961) chez Goytisolo et de Diego, qu'elle supplie de l'emmener en voiture avec lui jusqu'en Provence, alors qu'ils sont en pleine crise sentimentalo-amoureuse et bientôt sexuelle ${ }^{16} . .$. On notera un point important, c'est que Les platanes date de 1960, soit plus de vingt ans avant Les cabines de bain qui est encore plus autobiographique (ce n'est pas par hasard si Les platanes est encore écrit à la troisième personne), les points de convergence entre la « vraie vie » de Lange et Goytisolo étant davantage cachés sous le masque de la fiction dans ce récit précoce.

De plus, si Les cabines de bain faisait le point après plus de vingt-cinq ans d'histoire commune, Les platanes appartient à une époque où le coming out (révélation publique de son homosexualité) de Goytisolo n'avait pas été faite, pas même en privé (cf. l'autobiographie de Goytisolo Coto vedado et la lettre à Monique qui y est reproduite), ce qui modifie tout de même les perspectives. Ici, point de référence claire à l'homosexualité. Par rapport à Señas de identidad, on dira donc que ce pourraient être les réactions de la Dolores d'avant ce roman, qui ne connaîtrait pas encore la « différence » de son mari, et sur laquelle en revanche, vingt ans après, elle reviendra abondamment dans Les cabines de bain.

Il existe certes un récit - le seul - où, dès 1962, Monique Lange sous-entend davantage de choses sur la sexualité que les pensées de sa narratrice à la première personne, Sara, sur son compagnon, Miguel. Ces rapports sont de toute façon empreints d'une certaine difficulté liée, déjà, à une attirance quelque peu équivoque pour les hommes (même si rien n'est clairement dit). Citons par exemple cet extrait, parmi maints autres de la même valeur, où Sara s'adresse à son compagnon :

$\mathrm{Tu}$ avais tellement peur de ces hommes que tu commençais à aimer que tu buvais du vin pour les approcher. (...) Tandis que tu buvais, j'étais ivre de jalousie. Plus le vin se mêlait à votre sang, plus vous vous touchiez. Le rire devenait un prétexte pour vous toucher. (...) Pour les rejoindre, tu m'aurais donnée. Tu ne m'aimais que si je leur plaisais. (...) Et puis ils sentaient bon. Quand je rentrais avec toi, je respirais sur ton corps l'odeur des autres hommes que tu avais respirés ${ }^{17}$.

21 Cette intuition (?), car Señas de identidad de Juan Goytisolo ne date, rappelons-le, que de 1966, apporte un éclairage différent sur les « difficultés » du Diego des Platanes, datant de deux ans auparavant, et confirme notre analyse de l'intertextualité entre les deux écrivains, d'une œuvre à l'autre. La plage espagnole anticipe en cela et corrobore par avance, dans une perspective quasi borgésienne, le " grand déballage » des Cabines de bain, exactement vingt ans plus tard. 

protagoniste masculin, chez Goytisolo. La preuve est donnée par la narratrice, alors que le narrateur, évidemment, est censé ne jamais pénétrer dans l'esprit de sa femme, Dolores, par convention d'illusion "auto-biographique $»^{18}$. Et c'est ce qui fait que le critique est bien heureux d'avoir ces lignes croisées d'un auteur à l'autre sur ce que pense l'autre et sur ce qu'il/elle pense de l'autre; en effet, à une parole de Diego ( « - Il est tard. Ne rends pas les choses plus difficiles. Peut-être que rien n'est fini entre nous»), elle répond cyniquement (lucidement?): «-Sans doute, puisque rien n'a commencé » (p.74), mais on est encore dans le domaine de l'hétérosexualité, puisqu'il s'agit ici d'un problème de maîtresse (Nathalie). Les réflexions de Claudia nous révèlent un état d'esprit entre persistance de l'amour et désillusion. qui se refuse à faire l'amour. La femme tient très souvent le rôle actif, comme si le mâle ne pouvait que se laisser faire : "Elle se rappela une forêt. C'était l'automne. Elle avait violé Diego. Les feuilles crissaient sous leur corps » (p. 92), ou encore :

- Embrasse-moi (dit Claudia).

- Tu es saoûle?

- Non, mais tu me plais (...).

- Moi je ne sais pas encore bien ce qui me plaît vraiment en toi. /(...)/ Tu sais, dit-il, que j'aurais pu t'aimer.

- Tu aurais pu, mais tu ne peux pas ${ }^{19}$.

Cette citation est d'ailleurs à rapprocher de celles proposées pour l'illustration des rapports difficiles, voire aporistiques, entre Dolores/Claudia et Alvaro/Diego. Dans Señas de identidad, Dolores rappelle à Alvaro une certaine soirée sur une plage où le refus qu'il lui opposa de faire l'amour avec elle avait motivé cette scène, à rapprocher évidemment des Platanes :

- Al volver de la presa pasamos junto a una playa de arena y yo me quise bañar desnuda.

- Es posible.

- Llevábamos varias horas sin hablarnos porque la noche antes no quisiste hacer el amor conmigo. Tu dichoso Jumilla me había excitado $\mathrm{y}$, al acostarnos, me rechazaste con brusquedad.

- Excelente tu memoria. En efecto, así fue.

- Me dijiste : si tantas ganas tienes baja a la calle y búscate un hombre.

- Es lo que hiciste, $i$ no ? ${ }^{20}$.

Comme dans le cas d'Alvaro et de Dolores dans Señas, les relations du couple ne tardent pas à devenir franchement problématiques, comme on va le voir, ce que le lecteur ne peut s'empêcher d'interpréter pour le Diego des Platanes sur la base des tendances homosexuelles d'Alvaro, mais encore faut-il savoir qu'Alvaro est la matérialisation d'un Diego devenu ce qu'il est, chacun des deux Diego/Alvaro étant, de plus et surtout, le double à peu près d'un écrivain appelé Juan Goytisolo, compagnon d'un autre écrivain appelé Monique Lange, et tous deux narrant des morceaux de leur vie (quasiment toujours de leur propre point de vue, comme on l'a vu plus haut, ce qui fait l'intérêt de cette étude de l'intertextualité). Bien sûr, on peut trouver des prodromes de cette homosexualité latente soit dans La plage espagnole, comme on l'a vu plus haut, soit, de manière un peu plus subtile, dans les attitudes précises que Claudia prête à Diego, ou dans les dialogues qu'elle est censée retranscrire : 
(...) Si on savait ce qu'il y a dans la tête et dans le cœur des femmes, on ne pourrait pas les approcher. /(...)/ Tu m'en apprends sur la saloperie. Vous êtes doubles, fausses, ignobles, infâmes ${ }^{21}$. reconnaît dans un passage long et pessimiste que, malgré tout, et malgré cette « complicité » souhaitée au-delà de la chair, Dolores et lui sont devenus « dos extraños, asombrado cada uno con su ignorancia de la vida del otro, algo desamparados también por el derrumbe de todos los proyectos, quimeras e ilusiones $»^{25}$ (p. 297). 
sent aussi la même ambiguïté des désirs dans le passage de Hermann, le jeune éphèbe allemand pris en auto-stop par le couple des Platanes (pp. 126-129), que dans Para vivir aquí, Fin de fiesta ou La isla et les histoires graveleuses à trois qui y sont racontées (entre un pêcheur et une belle touriste nordique de passage sur la côte espagnole, la plupart du temps, mais aussi entre compatriotes). Ici, dans Les platanes, Claudia tente de séduire le jeune auto-stoppeur, lui caresse le genou, tandis qu'elle lui dit que Diego et elle sont frère et sœur, tout en embrassant et en caressant son compagnon. Diego, loin d'être jaloux, trouve gentil le jeune Allemand et insiste pour le garder dans la voiture, puis pour débarquer Claudia et son protégé dans un champ... Outre la perversité assumée de la situation, on retiendra ce dialogue, sinon équivoque et énigmatique, du moins prophétique, lorsque Hermann prend peur devant les assauts de Claudia (ce qui ramène aussi à l'idée du viol d'un homme par une femme) :

- Il est peut-être pédéraste, suggéra-t-elle.

- Chaque fois que tu fais peur à un homme, tu crois qu'il est pédéraste.

- Non, dit Claudia, avec toi je suis habituée, mais tu sais, en Allemagne, c'est connu, les hommes s'adorent entre eux ${ }^{26}$.

En ce qui concerne la différence sexuelle, la narratrice des Cabines de bain insiste avec raison sur la rivalité qu'elle a connue, en tant que femme, face aux Arabes - des Arabes parias, pauvres, ce qui fait le lien avec les deux catégories que l'on a indiquées auparavant :

Lui, le fils de riches Espagnols, n'avait pu toucher le fond de l'amour - ou de ce que l'on peut prendre pour l'amour - qu'en rejoignant des hommes qui avaient les mains abîmées par la société dont il était issu. (...)

La jeune femme, qui était consciente de la marge de perversité qu'il pouvait y avoir chez un écrivain à n'aimer que des analphabètes, lui était reconnaissante de lui avoir fait entrevoir ce monde-là (... ${ }^{27}$.

Le lecteur de Goytisolo connaît cet amour à la Jean Genet pour les parias, sur lequel le romancier jette un éclairage cette fois on ne peut plus cru - le livre a fait scandale en Espagne - dans cette auto-fiction, la dernière à ce jour, qu'est Carajicomedia (2000, non traduit à ce jour ${ }^{28}$ )

Dans Señas de identidad, roman sur lequel on a choisi, donc, de se pencher plus particulièrement dans cette étude, cet amour qui n'ose pas dire son nom envers un paria se fait jour dès l'adolescence d'Alvaro, en la personne de l'ouvrier paysan nommé Jerónimo, auquel l'auteur consacre plus de six pages (et de multiples références tout au long de ce même roman). Lange, pour sa part et sur le même sujet, évoque une scène où, alors qu'elle était enceinte, elle avait reçu des compliments moqueurs d'ouvriers, et compare cet épisode avec ce qui lui arriverait des années après : «Vingt ans plus tard, d'autres ouvriers, dans le même caniveau, des immigrés aux mains calleuses, seraient ses rivaux » (pp. 64-65). Elle perce à jour, peut-être un peu tard, mais elle le fait tout de même sans se voiler la face, le " secret " goytisolien de cette attirance envers les parias. Les scènes ou réflexions ambiguës sur ce sujet se multiplient dans Señas de identidad car elles n'ont pas toutes la clarté originelle du trick (rencontre sexuelle occasionnelle, furtive et le plus souvent anonyme) avec l'Arabe dans la rue. Cette scène de l'Arabe modifie donc définitivement les données. Juste après l'évocation par Alvaro de sa vie commune avec Dolores (cf. plus haut le «dialogue imaginaire ») en termes encore quelque peu sybillins, a lieu la rencontre du narrateur avec cet ouvrier maghrébin, marié (à deux femmes !) et père de six enfants, et dont on comprend qu'ils font l'amour, scène qui sera glosée par Monique Lange, même si ce n'est pas exactement celle-là, 
lorsqu'elle parle des rencontres de son compagnon avec des ouvriers dans le hammam tous les dimanches. Goytisolo insiste sur l'aspect brut, rude et très viril de l'ouvrier, comme Monique Lange le fera également :

El Arabe ha abandonado la contemplación del panorama de las gras y suelta a andar a su vez, cauto y receloso, con las manos hundidas en los bolsillos. A una veintena de metros de él puedes observar a tus anchas sus botas de goma, los pantalones de burdo azul mahón, la zamarra de cuero con las solapas forradas de piel, el pasamontañas de lana ceñido a la cabeza. Su presencia discreta gobierna la calle ${ }^{29}$.

Bien sûr, Goytisolo, surtout en 1966 avec ce roman coming out et néanmoins subtil, décide de ce qu'il écrit, mais Lange, rappelons-le, le pousse dans ses retranchements en le jugeant et en donnant sa position sur la manière dont elle-même, via ses héroïnes, a vécu cette relation, « de l'autre côté ». On a donc droit à deux visions des choses grâce à cette intertextualité. En ce qui concerne spécifiquement la sexualité, ici l'homosexualité du compagnon, elle apparaît enfin explicitement, toujours dans le cadre d'une découverte du monde arabe :

Ensuite, comme les touristes de tous les pays du monde, de tous les voyages du monde, ils marchandaient des djellabas, des colliers, des babouches. (...)

Bien avant qu'elle ne sache qu'il était homosexuel, bien avant qu'il ne le sache, lui aussi, elle aimait marchander ${ }^{30}$.

On remarquera particulièrement qu'elle évoque le caractère non connu, pendant longtemps, de sa propre homosexualité par son mari/compagnon, ce qui nous rend tout de même moins suspicieux quant à la possible hypocrisie de l'auteur concernant ses désirs (savait-il que son attirance pour les Arabes et/ou les parias était aussi, même si ce n'était pas exclusivement, sexuelle?).

Sa compréhension est telle qu'elle accepte même de parler avec lui de ses relations avec les hommes :

Un dimanche - ça se passe toujours le dimanche parce que les ouvriers ne travaillent pas le dimanche - il est revenu très beau avec un visage pur et lisse. Il avait été au hammam puis faire l'amour avec un Algérien. Elle avait été bouleversée par la beauté (morale aussi) qui se dégagait de lui ce jour-là ${ }^{31}$.

Il s'agit ici, comme on l'a annoncé plus haut, sinon de l'épisode célèbre de Señas de identidad dont nous avons parlé, du moins de ce type d'épisodes, de tricks qui se répètent semble-t-il à l'envi. On remarque que, même si la capacité de réflexion sur cette attirance de son compagnon envers les Arabes ne souffre pas, chez la narratrice, de masque ou de faux-semblant, bref, qu'elle ne lui passe rien, elle semble également comprendre la portée éthique d'une telle attirance.

Un autre point de concorde entre ces deux littératures, c'est le temps que cela a pris à l'un et à l'autre d'accepter, l'un cette différence pour lui-même (car entre 1954 et 1966, les romans de Goytisolo, reflet de sa trajectoire vitale, ne parlent pas explicitement, et pour cause, de cette attirance encore latente, encore taboue, pour les hommes, des hommes d'un certain type : rude et pauvre), l'autre cette différence de son compagnon : "En dépit de son goût de la confidence, ça lui avait bien pris quelques années avant d'être capable de raconter le chemin parcouru " (p. 69). Mais enfin, p. 303 de Señas de identidad, Alvaro a cette révélation après sa syncope. C'est quasiment une synthèse et une conclusion que propose Alvaro/Goytisolo, mais aussi un point de départ pour sa nouvelle vie avec ce passage de l'évanouissement. Laissons-le alors parler, en remarquant (nous l'avons souligné) que l'attirance est d'abord sexuelle, comme l'a aussi noté Lange : 
Los viejos que agonizaban sin familia, los obreros amputados por sus propios útiles de trabajo, los árabes y negros que, allah yaouddi, se lamentaban en idioma para ti incomprensible te habían mostrado el camino por el que un día u otro tenías que pasar si querías devolver, limpio, a la tierra, lo que en puridad le pertenecía. Tu salvación debías buscarla allí, en ellos y su universo oscuro, como de instinto y sin aprendizaje de nadie, severamente, junto a ellos, habías buscado el amor ${ }^{32}$.

En conclusion, il nous semble que Monique Lange n'a jamais aussi bien résumé le rapport ambigu et complexe que Goytisolo entretient, littérairement bien entendu..., avec les Arabes, le sexe et la politique, que de cette manière :

(...) (L)orsqu'elle faisait rire ses amis en disant: «Il a résolu sexuellement ses problèmes politiques », elle savait très bien ce qu'elle voulait dire et puisqu'il lui avait fallu organiser sa douleur, elle avait décidé qu'elle souffrait selon sa morale et que, s'il avait aimé des affreux bourgeois, c'est alors qu'elle aurait eu honte ${ }^{33}$.

Ce qui importe ici, avec cette intertextualité que nous avons qualifiée malicieusement de "conjugale ", c'est que Monique Lange a opéré comme un décryptage des mots de Goytisolo. Et il s'agit pour le lecteur et pour le chercheur d'un décryptage lucide dans des personnages et des œuvres qui sont, malgré tout, de fiction : loin de nous l'idée de proposer une équivalence absolue Alvaro = Juan Goytisolo et Claudia = Monique Lange, par exemple. Ce pourraient être - et d'ailleurs c'en sont, par la grâce de la littérature des êtres de fiction. Tout se passe alors comme si un autre écrivain nous révélait l'envers du décor et les motivations profondes de Julien Sorel ou de La Regenta (de Clarín), de l'Ariane de Belle du Seigneur ou de la femme de Mario dans Cinco horas con Mario (de Miguel Delibes). Evidemment, il ya un intérêt supplémentaire à penser que se meuvent des ombres réelles derrière ces personnages, mais finalement, ce n'est pas cet aspect-là qui est le plus intéressant...

Pour terminer, nous aimerions dire que le dernier « roman » de Goytisolo, Carajicomedia qui, comme beaucoup de livres du romancier espagnol, depuis le début des années 80 , joue avec la réalité et la fiction, et particulièrement avec le statut/la statue de l'écrivain «Juan G. " (l'écrivain espagnol s'étant toujours amusé de son " personnage » dans ses nombreuses auto-fictions), apporte des preuves définitives à la vision qu'avait Monique Lange des obsessions goytisoliennes, comme si, à soixante-dix ans, Juan Goytisolo "lâchait les chiens", avec distance et humour sur lui-même, et en se moquant totalement de ce que peuvent penser les autres. Gageons qu'il souhaite surtout continuer à «épater le bourgeois " avec ses révélations (dans un ton certes plus cru encore que celui de Lange) sur ses amours de pissotières. Mais à propos de crudité, laissons une dernière fois la parole à Monique Lange qui prouve qu'elle avait bien compris la problématique secrète du protéiforme Juan G./Alvaro/Miguel//Diego et de son rapport avec l'écriture :

Je te regardais faire la putain avec ces ouvriers. Tu voulais, au-delà de tout, être leur semblable, mêlé, mêlé. Tu voulais oublier que tu les quittais pour rentrer dans ta maison haute aux draps de fil. Même le linceul de ta mère était un drap de fil. Tu ne leur ressemblerais jamais, même si chacun de tes mots était un jour de leur travail, un jour de leur vie, même si chacun de tes gestes était une grève ${ }^{34}$. 


\section{NOTES}

1. Tous deux chez Gallimard, comme les autres œuvres de fiction de Monique Lange.

2. Nous citerons l'édition Mondadori (Barcelone) de 1991. La traduction des passages espagnols, non seulement de ce roman mais de tous les autres de Goytisolo, est faite par nos soins.

3. Cf. le remarquable ouvrage coordonné par Abdelatif BEN SALEM, Juan Goytisolo ou Les paysages d'un flâneur, Paris : Fayard/Institut du Monde Arabe, 1996 et la bibliographie la plus complète (dans plusieurs langues) disponible à ce jour sur Juan Goytisolo, et Emmanuel LE VAGUERESSE, Juan Goytisolo, Ecriture et marginalité, Paris : L'Harmattan, 2000. On y trouvera également des réflexions sur l'intertextualité intra-goytisolienne, elle aussi abondamment pratiquée par l'auteur espagnol.

4. Les cabines de bain, pp. 100-101.

5. Op. cit., p. 143.

6. « Le trachome a dévoré les yeux des vendeurs de billets de loterie qui promettent 'la chance pour aujourd'hui' (...) », La Chanca, Paris : Librairie Espagnole, 1962 (éd. citée 1981), p. 14.

7. « Le chemin s'ouvre à travers la terre en friche et Escuyos surgit, soudain, sur la droite. C'est un village misérable, ravagé par les ouragans, et dont les maisons poussent à tort et à travers. Il n'y a pas de rues, ni même de chemins dignes de ce noms ", Campos de Nijjar, Barcelone : Seix Barral, 1960 (éd. citée 1979), p. 93.

8. « Lors de ton premier contact avec le Sud la vitalité rude et sauvage de ces hommes te captivait (au point d'énerver Dolores) ", Señas de identidad, p. 140. La parenthèse est bien évidemment dans le texte original.

9. «Assis au bord du chemin, un homme vêtu d'une chemise militaire, se caressait les joues, couvertes d'une barbe de plusieurs jours » et «Victorino (...) m'aida à me mettre au lit. Il s'était assis à côté de moi et me regardait avec une expression voisine de l'amour ", La Chanca, p. 61 et p. 93.

10. « ... Ton premier contact avec le Sud avait eu lieu au travers de ses hommes. Depuis tout petit, tu les reconnaissais grâce à leur langage et à leur façon de parler, si différents de ceux des Catalans. Tu les entendais chanter sur les échafaudages, blasphémer dans les tranchées des chantiers, discuter pendant qu'ils balayaient les rues, discuter le coup au soleil (...) », Señas de identidad, p. 313.

11. Les cabines de bain, p. 54 .

12. « virilité et rudesses thérapeutiques que, des mois auparavant, tu avais trouvées chez des noirs et des mulâtres de La Havane, poursuivi par un amour adolescent (l'ouvrier agricole Jerónimo) qui était comme le présage de la syncope du Boulevard Richard Lenoir et de ta révocable existence à crédit », Señas de identidad, p. 37.

13. Les cabines de bain, p. 99.

14. « éloigne-toi de ton troupeau ta déviance t'honore/tout ce qui te sépare d'eux cultive-le/ce qui les gêne en toi glorifie-le/négation stricte absolue de leur ordre voilà ce que tu es ", Señas de identidad, pp. 348-350.

15. Les cabines de bain, p. 140. La parenthèse est dans le texte original.

16. Il faut dire ici qu'il existe une intertertextualité multiple entre tous les romans et récits de Goytisolo et ceux de Lange publiés dans les années 60 , ne serait-ce que thématiquement, dans ces histoires de bourgeoisie bohême frottée d'engagement à 
gauche qui peut, comme ici ce couple ou comme ceux de La isla, de Para vivir aquí (Pour vivre ici, 1960, éd. française 1962) ou de Fin de fiesta (Danses d'été, 1962, éd. française 1964), décider de rouler ensemble vers le Sud sur un coup de tête...

17. La plage espagnole, Paris : Gallimard, 1962, pp. 72-74. « Ces hommes que (son compagnon) commençai( $t$ ) à aimer », ce ne sont pas « les hommes » en général mais les ouvriers, les pêcheurs, les gens de peu : pourtant, l'expression est ambiguë à souhait et ce n'est sûrement pas un hasard sous la plume de Monique Lange.

18. Sauf, au début des années soixante, l'un des récits - le troisième - de Fin de fiesta où la voix narrative est féminine et, de manière exceptionnelle, juge l'attitude de son mari par rapport aux pauvres et aux parias : « Juan parecía feliz en medio de aquella desolación. Su vitalidad renacía al contacto de la miseria y las pupilas le brillaban como en la tarde en que me llevó a Montjuich y se emborrachó con los hombres » («Juan avait l'air heureux au beau milieu de cette désolation. Sa vitalité renaissait au contact de la misère et ses pupilles brillaient comme le soir où il m'avait emmenée à Montjuich et où il s'était saoulé avec les hommes »), «Tercera », Fin de fiesta, pp. 72-73.

Curieusement, Goytisolo anticipe, ici avec beaucoup de recul (ou retrouve, puisque Fin de fiesta est publié la même année que Les platanes, en 1962), les propres paroles de Lange sur les différents doubles fictionnels de son compagnon. Ceci dit, ce récit de Fin de fiesta est moins ambigu sur la sexualité de cet homme (qui se nomme, en plus, Juan...) que La plage espagnole, comme on l'a vu plus haut.

19. Les platanes, p. 115.

20. «- En revenant de la digue, on est passés près d'une plage de sable fin et j'ai voulu me baigner toute nue./ - C'est possible./ - Ca faisait plusieurs heures qu'on ne se parlait pas parce que la nuit précédente tu n'avais pas voulu faire l'amour avec moi. Ton maudit Jumilla m'avait excitée et, quand on s'est couchés, tu m'as repoussée brusquement./ - Tu as une très bonne mémoire. En effet, c'est comme ça que ça s'est passé./ - Tu m'as dit : si tu en as tellement envie, sors dans la rue et trouve-toi un mec./ - C'est ce que j'ai fait, non? ", Señas de identidad, pp. 100-101.

21. Les platanes, pp. 136-137.

22. «Dolores était apparue à la porte de la galerie. (...) En se croisant, vos regards ressuscitaient l'espace de quelques instants la douce impression de jeu et de complicité d'autrefois : croyance illusoire d'une unité morale que ni le temps ni l'humaine pourriture ne détruiraient ", Señas de identidad, p. 37.

23. « Mes papiers d'identité sont faux ».

24. «- (...) Quand je devine le désir contre lequel je ne peux pas lutter».

25. « deux étrangers, chacun étant étonné par son ignorance de la vie de l'autre, quelque peu désamparés aussi par l'effondrement de tous les projets, chimères et illusions ».

26. Les platanes., p. 128.

27. Les cabines de bain, pp. 64-65.

28. « titre-jeu de mots intraduisible sur la paronymie entre Tragicomédie et Comédie du " carajo » = pénis, en espagnol.

29. «L'Arabe a cessé de contempler le panorama des grues et se met à marcher à son tour, méfiant et soupçonneux, les mains enfoncées dans les poches. A une vingtaine de mètres de lui, tu peux observer à ton aise ses bottes en caoutchouc, le pantalon taillé dans un coton bleu grossier, la pelisse en cuir aux revers doublés, le passe-montagne en laine bien ajusté à la tête. Sa présence discrète gouverne la rue ", Señas de identidad, p. 281. 
30. Les cabines de bain, p. 75. Nous soulignons pour l'aspect qui seul nous intéresse ici, curieusement lié d'ailleurs au « marchandage »...

31. Op. cit., p. 68.

32. «Les vieux qui agonisaient sans famille, les ouvriers amputés par leurs propres outils de travail, les Arabes et les Noirs qui, allah yaoudi, se plaignaient dans une langue pour toi incompréhensible, t'avaient montré le chemin par où un jour ou l'autre tu devais passer si tu voulais restituer, proprement, à la terre, ce qui clairement lui appartenait. Ton salut tu devais le chercher là-bas, en eux et dans leur univers sombre, comme instinctivement et sans apprentissage de personne, sévèrement, près d'eux, tu avais cherché l'amour ", Señas de identidad, p. 303. La ponctuation si spéciale de Goytisolo a été respectée.

33. Les cabines de bain, p. 45.

34. La plage espagnole, p. 73.

\section{RÉSUMÉS}

Juan Goytisolo (né en 1931), écrivain espagnol, et Monique Lange (1926-1996), écrivain français, ont produit chacun une œuvre à forte résonance autobiographique, sous couvert de fiction, et qui crée un effet très particulier d'intertextualité par le simple fait qu'ils étaient, dans la vie, mari et femme... D'où ce titre "d'intertextualité conjugale ", car ces récits auto-fictionnels croisés de relations communes s'enrichissent particulièrement du regard critique et lucide de la romancière sur les obsessions personnelles de son écrivain de mari, les décryptant par le biais de personnages « inventés » qui, non seulement renseignent sur un destin littéraire partagé, mais encore et surtout, dans une configuration rare de double écriture, permettent chez Lange de lire différemment les lignes souvent floues (sur la sexualité, les parias, le monde arabe) de l'écrivain espagnol bien connu, et même de démystifier ses discours d'Espagnol marginal.

\section{AUTEUR}

\section{EMMANUEL LE VAGUERESSE}

Université de Reims Champagne-Ardenne 